\title{
Estratégia\&Negócios
}

ISSN 1984-3372

http://www.portaldeperiodicos.unisul.br/index.php/EeN/

\section{MEIOS DE COMPARTILHAMENTO DO CONHECIMENTO NO CONTEXTO DE PROJETOS DE TECNOLOGIA DA INFORMAÇÃO}

\section{MEANS OF KNOWLEDGE SHARING IN INFORMATION TECHNOLOGY PROJECTS}

\section{Clarissa Carneiro Mussi}

Doutora em Administração, Mestre em Administração e Bacharel em Ciência da Computação. Professora do Programa de Pós-graduação em Administração da Universidade do Sul de Santa Catarina.

E-mail: mussi.clarissa@gmail.com

\section{Maria Terezinha Angeloni}

Doutora em Administração pela Université Pierre Mendes France. Mestre em Administração pela Université Pierre Mendes France e pela Universidade Federal da Paraíba. Graduada em Administração pela Universidade Federal de Santa Catarina - UFSC.

E-mail: angelggc@hotmail.com

Recebido em 08/09/2011. Aprovado em 26/10/2011. Disponibilizado em 03/12/2011. Avaliado pelo Sistema double blind review

R. eletr. estrat. neg., Florianópolis, v.4, n.2, p.30-60, jun./dez. 2011 http://portaldeperiodicos.unisul.br/index.php/EeN/index
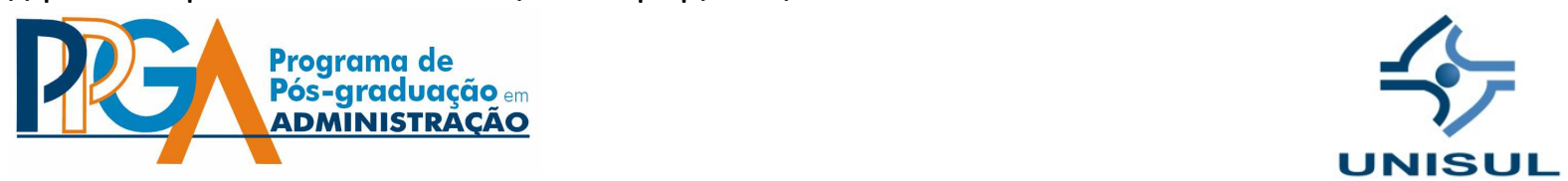

CCopyright 2008 UNISUL-PPGA/Estratégia e Negócios. Todos os direitos reservados. Permitida citação parcial, desde que identificada a fonte. Proibida a reprodução total. Em caso de dúvidas, consulte o editor: ademar.unisul@gmail.com; (48) 3229-1932. 


\section{MEIOS DE COMPARTILHAMENTO DO CONHECIMENTO NO CONTEXTO DE PROJETOS DE TECNOLOGIA DA INFORMAÇÃO \\ Clarissa Carneiro Mussi - Maria Terezinha Angeloni}

\section{RESUMO}

Este estudo buscou investigar de que forma se configurou o compartilhamento do conhecimento entre as partes envolvidas no projeto de implementação de um sistema ERP (SAP R/3) em uma instituição de ensino superior de Santa Catarina/Brasil. Especificamente, o trabalho investigou os meios de compartilhamento do conhecimento utilizados, seus objetivos e sua influência no projeto em questão. Considerando os objetivos propostos, a abordagem desta pesquisa é qualitativa do tipo estudo de caso. Os dados foram coletados por meio de entrevistas semiestruturadas, questionários abertos e análise documental. $\mathrm{O}$ tratamento $\mathrm{e}$ interpretação dos dados são predominantemente qualitativos. Dentre as principais conclusões obtidas pode-se citar a influência significativa do compartilhamento do conhecimento no projeto de implementação do sistema. Foi observada a utilização de vários meios de compartilhamento tanto do conhecimento explícito quanto tácito. Estes meios propiciaram modos de conversão do conhecimento. Finalmente, a análise dos dados conduziu ao entendimento de que a definição dos meios pelos quais o conhecimento é compartilhado e a forma com que esses meios são utilizados podem facilitar ou dificultar o compartilhamento e, consequentemente, a implementação do sistema.

Palavras-chave: Tecnologia da informação. Compartilhamento do conhecimento. ERP. Projetos.

\section{INTRODUÇÃo}




\section{MEIOS DE COMPARTILHAMENTO DO CONHECIMENTO NO CONTEXTO DE PROJETOS DE TECNOLOGIA DA INFORMAÇÃO \\ Clarissa Carneiro Mussi - Maria Terezinha Angeloni}

O reconhecimento dos ativos intangíveis como recursos estratégicos que necessitam ser gerenciados tem conduzido a uma crescente atenção à gestão do conhecimento nas organizações em geral e no contexto da gestão de projetos em particular. De acordo com Kasvi, Vartiainem e Hailikari (2003), o sucesso da gestão de um projeto é baseado no conhecimento acumulado e nas competências individuais e coletivas. Como observam Karapetyan e Otieno (2011), o número crescente de pesquisas sobre aspectos da gestão do conhecimento em ambientes de projeto evidencia o interesse da comunidade científica nestas duas áreas.

O objetivo principal da gestão de um projeto centra-se em combinar bases de conhecimento dos integrantes do time do projeto e demais stakeholders visando a alcançar o sucesso do projeto (KARAPETYAN; OTIENO, 2011). Entretanto, a gestão do conhecimento no contexto de projetos enfrenta vários desafios, considerando a natureza de um projeto: os projetos envolvem pessoas com diferentes conhecimentos, culturas e linguagens; são limitados a um período de tempo e as pessoas envolvidas e as lições aprendidas são freqüentemente dispersas quando o projeto termina; algumas pessoas não se envolvem com o projeto do início ao fim (BRESNEN et al, 2003, KASVI; VARTIAINEN; HAILIKARI, 2003, KARAPETYAN; OTIENO, 2011). Torna-se, portanto, difícil desenvolver um processo sistemático de forma a maximizar o fluxo de conhecimento e a aprendizagem.

Projetos de tecnologia da informação (TI), foco deste artigo, podem ser o projeto de desenvolvimento e implementação de um novo produto, serviço ou processo (KARLSEN; GOTTSCHALK, 2004). O projeto de TI examinado neste estudo consistiu da implementação de um sistema ERP (Enterprise Resource Planning) em uma Instituição de Ensino Superior. As questões críticas relativas a esses sistemas repousam em seu projeto de implementação, especialmente na mudança da tradicional concepção departamental para uma visão centrada em processos, e na dificuldade das organizações de alinhar os imperativos tecnológicos do sistema às necessidades de negócio (DAVENPORT, 1998, WANG et al., 2007). Esse alinhamento 


\section{MEIOS DE COMPARTILHAMENTO DO CONHECIMENTO NO CONTEXTO DE PROJETOS DE TECNOLOGIA DA INFORMAÇÃO \\ Clarissa Carneiro Mussi - Maria Terezinha Angeloni}

requer conhecimento dos processos organizacionais críticos e conhecimento detalhado do sistema (SOH; KIEN; TAY-YAP, 2000).

Como ressaltam Clegg, Waterson e Axtell (1997), o projeto de implementação de um sistema é "intensivo em conhecimento", incorporando a expertise e as habilidades de muitas pessoas. Mais especificamente, o projeto de implementação de um sistema ERP envolve a interação de grupos de indivíduos com conhecimentos distintos e específicos: por um lado, a empresa representada por seus colaboradores com conhecimento das necessidades organizacionais e da infraestrutura de tecnologia existente; por outro, os fornecedores e/ou consultores do sistema com conhecimento de sua funcionalidade e experiência vivenciada na sua implementação.

Enquanto a interação freqüente e a solução de problemas em conjunto traduzem-se na maneira lógica para que os diversos conhecimentos sejam compartilhados, a variedade de background e os diferentes interesses das partes envolvidas tornam difícil alcançar a integração desse conhecimento (SOH; KIEN; TAYYAP, 2000, TESCH et al. 2009). Além disso, muito do conhecimento é tácito, o que dificulta ainda mais seu compartilhamento (VANDAIE, 2008).

Em decorrência da natureza diversa dos conhecimentos envolvidos em um projeto de tecnologia da informação, torna-se necessário considerar o compartilhamento e a integração desses conhecimentos de forma a contribuir para a efetividade do projeto (CLEGG; WATERSON; AXTELL, 1997, SOH; KIEN; TAY-YAP, 2000, MABERT, 2001, VANDAIE, 2008, TESCH et al., 2009). Baseando-se em pesquisas empíricas, Mabert (2001) ressalta que uma das características das empresas que obtiveram sucesso em projetos ERP residia no estabelecimento de um processo claro de compartilhamento de conhecimento dos consultores externos para a equipe interna de implementação visando a reter o conhecimento para a empresa. Como reforça Jones (2005), ironicamente, ao mesmo tempo em que um sistema ERP visa a melhorar o compartilhamento e integridade de informações e conhecimentos 
empresariais, as organizações que desejam implementá-lo precisam ter a capacidade de um compartilhamento efetivo do conhecimento.

O objetivo deste estudo está voltado à análise da forma como se configurou o compartilhamento do conhecimento entre as parte envolvidas no projeto de implementação de um sistema $\operatorname{ERP}(S A P / R 3)$ em uma Instituição de Ensino Superior. Em especial, à investigação dos meios de compartilhamento, tanto do conhecimento explícito quanto tácito, utilizados, seus objetivos e a sua influência no projeto.

Este artigo está estruturado em cinco seções. Esta primeira apresenta a introdução que delineia o tema, o contexto e os objetivos da pesquisa. A segunda seção, a seguir, contempla os fundamentos teóricos referentes ao tema que se pretende investigar, a terceira apresenta a metodologia pela qual se desenvolveu a pesquisa, a quarta se estrutura em torno da apresentação e análise dos dados coletados e por fim, a quinta seção expõe as considerações finais do estudo.

\section{BASE TEÓRICA}

\subsection{A GESTÃO DE PROJETOS DE TI E A GESTÃO DO CONHECIMENTO}

Um projeto pode ser definido como um empreendimento temporário com o objetivo de criar um produto ou serviço único (PMI, 2008). Ruuska e Vartiainen (2003) consideram um projeto um sistema de trabalho complexo onde elementos procedurais, organizacionais e humanos são integrados. A gestão de projetos é a aplicação de conhecimentos, habilidades e técnicas para projetar atividades que visem a atingir os requerimentos do projeto (PMI, 2008). 


\section{MEIOS DE COMPARTILHAMENTO DO CONHECIMENTO NO CONTEXTO DE PROJETOS DE TECNOLOGIA DA INFORMAÇÃO \\ Clarissa Carneiro Mussi - Maria Terezinha Angeloni}

Uma das competências críticas para a gestão de projetos é a gestão do conhecimento (RUUSKA; VARTIAINEN, 2003, SENG; ZANNES; PACE 2002, BRESNEN et al, 2003, CRAWFORD, 2000). A gestão do conhecimento no contexto de um projeto é a aplicação de princípios e processos projetados para tornar conhecimento relevante disponível ao time do projeto. A gestão do conhecimento efetiva facilita a criação e integração do conhecimento, minimiza a perda de conhecimento, e preenche lacunas de conhecimento ao longo do projeto.(REICH, 2007, p.8, tradução nossa). Como observam Kasvi, Vartiainen e Hailikari (2003), a gestão do conhecimento em projetos é fundamental se a organização quer se tornar uma organização de aprendizagem e ser capaz de aplicar os resultados e lições aprendidas de um projeto em outros projetos. Contudo, Bresne et al (2003) ressaltam que a gestão do conhecimento em projetos está diante de vários desafios, considerando as descontinuidades de fluxos de pessoas, materiais e informações características do contexto da gestão de projetos.

Kasvi, Vartiainen e Hailikari (2003) identificam quatro grupos de atividades relacionadas à gestão do conhecimento em um projeto: (1) criação do conhecimento, (2) administração do conhecimento (armazenagem, organização e recuperação), (3) compartilhamento do conhecimento e (4) utilização do conhecimento. Este artigo focaliza o compartilhamento do conhecimento.

Independentemente de quantas ou de quais fases um projeto compreenda, e de como essas fases se relacionam, o ponto chave é que vários indivíduos estão incorporando-Ihe diferentes formas de conhecimento, habilidades e expertise sob um período de tempo (CLEGG; WATERSON; AXTELL, 1997, p. 359). Em um projeto de TI, conhecimento da estratégia do negócio, da estratégia de tecnologia da informação, de análise e projeto de sistemas, de gerenciamento de projetos, são alguns exemplos. As bases de conhecimento e linguagens distintas dos indivíduos integrantes de um projeto podem tornar o efetivo compartilhamento do conhecimento mais problemático (BRESNE et al, 2003, TESCH et al. 2009). Criar um clima e mecanismos para o compartilhamento do conhecimento, aumentará a 
capacidade da equipe do projeto de alcançar o sucesso do projeto (KARLSEN; GOTTSCHALK, 2004). Mas, o que se entende por compartilhar conhecimento?

\subsection{O COMPARTILHAMENTO DO CONHECIMENTO EM PROJETOS DE TI}

O compartilhamento do conhecimento é caracterizado por Davenport e Prusak (1998) como a transferência do conhecimento, seja esta espontânea (informal) ou estruturada (formal), entre indivíduos. Os autores ressaltam que o termo transferência envolve duas ações: a transmissão (envio ou apresentação do conhecimento a uma pessoa ou grupo) e a absorção (incorporação ou assimilação desse conhecimento por quem o recebeu).

Para Lahti (2000), o compartilhamento do conhecimento envolve a transmissão e a difusão do conhecimento dentro de uma organização ou entre diferentes organizações. Observa-se que no contexto de projetos de $\mathrm{TI}$, os dois casos podem estar presentes. A equipe de um projeto de implementação de um sistema ERP, por exemplo, é geralmente composta pelo fornecedor e/ou consultores do sistema e pelos membros da própria organização. Além disso, o compartilhamento do conhecimento pode ocorrer dentro do projeto - entre seus integrantes - e fora do projeto - entre a equipe do projeto e a organização (KASVI; VARTIAINEM; HAILIKARI, 2003, VANDAIE, 2008). Integrantes da organização e consultores trazem para o projeto diferentes níveis de compreensão dos processos organizacionais existentes e do sistema a ser implementado, de forma que o fator crítico de sucesso não é meramente a reprodução de "melhores práticas", mas o grau de compartilhamento do conhecimento entre os participantes da implementação. (HUNG et al., 2011).

A dificuldade de compartilhar o conhecimento está diretamente relacionada ao tipo de conhecimento envolvido (explícito ou tácito). O conhecimento explícito pode ser codificado em procedimentos ou representado em documentos, livros, arquivos e banco de dados e, assim, identificado e compartilhado mais 
facilmente. Por outro lado, o conhecimento tácito (pessoal e subjetivo, incorporado à experiência individual ao longo do tempo) para ser compartilhado exige um intenso contato pessoal, seja a partir de uma parceria, uma relação de orientação ou aprendizado (DAVENPORT; PRUSAK, 1998, SVEIBY, 1998, NONAKA; VON KROGH, 2009).

Um projeto de TI é permeado tanto de conhecimento explícito quanto tácito que provêem das diferentes partes envolvidas no projeto. Considerar e identificar esses conhecimentos, possibilitando meios de compartilhamento coerentes com seu tipo, contribui para um compartilhamento mais efetivo (LAHTI, 2000, JASIMUDDIN, 2008). Meios de compartilhamento são aqui entendidos como recursos pelos quais o conhecimento pode ser compartilhado.

Karlsen e Gottschalk (2004), citando Argote et al (2000), abordam alguns meios de compartilhamento do conhecimento, muitos dos quais podem ser utilizados em projetos de $\mathrm{Tl}$, como por exemplo: treinamento, comunicação e observação de pessoal, tecnologia, rotinas de replicação, publicações e apresentações, interação com fornecedores e clientes, alianças e outras formas de relacionamentos interorganizacionais. Nonaka e Takeuchi (1997) sugerem a utilização de metáforas e analogias como um meio de facilitar o compartilhamento do conhecimento tácito.

Mussi (2002) reúne vários meios de compartilhamento do conhecimento que podem ser utilizados no projeto de implementação de um sistema ERP como: diálogo, discussão hábil, reflexão, argumentação e inquirição coletiva, metáforas e analogias, questionários e entrevistas, reuniões, workshops, visitas a outras empresas e os contatos com material e pessoas especializadas, treinamentos, simulações, documentos, repositórios de conhecimento e os manuais.

Os meios de compartilhamento do conhecimento podem contribuir para processos de geração e compartilhamento do conhecimento, chamados por Nonaka e Takeuchi (1997) de modos de conversão do conhecimento. 


\section{MEIOS DE COMPARTILHAMENTO DO CONHECIMENTO NO CONTEXTO DE PROJETOS DE TECNOLOGIA DA INFORMAÇÃO \\ Clarissa Carneiro Mussi - Maria Terezinha Angeloni}

\subsection{O COMPARTILHAMENTO E A CONVERSÃO DO CONHECIMENTO}

Nonaka e Takeuchi (1997) defendem a idéia de que o conhecimento humano é criado e expandido a partir da interação entre conhecimento tácito e explícito, a qual denomina de "conversão do conhecimento". Os autores apresentam quatro modos ou processos de conversão do conhecimento: socialização (tácito para tácito), externalização (tácito para explícito), internalização (explícito para tácito) e combinação (explícito para explícito).

Percebe-se que estes processos, embora tenham como finalidade primeira a criação do conhecimento envolvem o compartilhamento do mesmo, pode-se assim ressaltar a importância que o compartilhamento exerce na própria criação do conhecimento na organização. Para Nonaka e Takeuchi (1997), a criação do conhecimento acontece a partir da interação social entre indivíduos e não representa um processo confinado em um único indivíduo. Quinn, Anderson, e Finkelstein (1998) corroboram esta idéia quando dizem que o conhecimento cresce exponencialmente quando o compartilhamento é propriamente estimulado, pois este conduz a feedbacks, amplificações e modificações do conhecimento existente.

A socialização corresponde à troca de conhecimento tácito entre indivíduos, principalmente por meio do compartilhamento de experiências vivenciadas. O processo de socialização não necessariamente precisa fazer uso da linguagem para acontecer, pode se dar, por exemplo, por meio da observação, imitação e treinamento prático.

A externalização acontece quando o conhecimento tácito, difícil de ser comunicado e formulado, torna-se explícito, transmissível e articulável. A utilização de metáforas ou analogias é bastante eficaz no processo de externalização, pois permite aos indivíduos revelar o conhecimento tácito que de outra forma seria difícil de comunicar. 
A internalização é o processo de conversão do conhecimento explícito em conhecimento tácito por meio da verbalização e diagramação do conhecimento sob a forma de documentos, manuais ou histórias orais. O aprendizado da leitura de documentos ou manuais constitui-se um exemplo de internalização.

A combinação refere-se à troca e união de diferentes conhecimentos explícitos para gerar um conhecimento explícito novo. Pode ocorrer, por exemplo, por meio de documentos, reuniões, redes de computador e envolver a análise, a classificação, o acréscimo, a categorização.

Os quatro modos de conversão do conhecimento possibilitam a interação entre o conhecimento tácito e o conhecimento explícito permitindo que conhecimentos sejam gerados e compartilhados.

\section{METOdOLOGIA DE PESQUISA}

Dada a natureza do problema que se pretende investigar e os objetivos propostos, a abordagem desta pesquisa é qualitativa (STRAUSS; CORBIN, 2008) do tipo estudo de caso (YIN, 2009). A organização em cujo contexto o estudo empírico foi realizado trata-se de uma instituição de ensino superior do estado de Santa Catarina, a primeira Universidade do Brasil a implementar o ERP, conhecido como SAP R/3, da fornecedora alemã SAP.

A Universidade pesquisada conta com aproximadamente 28.000 alunos, 1.800 professores e 700 técnicos. No caso deste estudo, o universo da pesquisa corresponde às pessoas envolvidas direta e indiretamente no projeto de implementação do SAP R/3 na Universidade, abrangendo os seus três campi e as áreas desses campi relacionadas aos módulos do sistema implementados. 


\section{MEIOS DE COMPARTILHAMENTO DO CONHECIMENTO NO CONTEXTO DE PROJETOS DE TECNOLOGIA DA INFORMAÇÃO}

Clarissa Carneiro Mussi - Maria Terezinha Angeloni

Consideram-se como pessoas diretamente envolvidas aquelas que foram definidas formalmente pela instituição como constituintes do projeto (usuários-chave, representantes da área de $\mathrm{TI}$, etc.), além dos consultores do sistema SAP/R3, e como pessoas indiretamente envolvidas, os colaboradores da institutição que não participaram diretamente do projeto, mas que utilizam o sistema, chamados neste estudo de usuários-finais.

As pessoas diretamente envolvidas no projeto perfazem um total de trinta e sete (37) entre as quais estão pessoas da Universidade e pessoas externas (empresas contratadas). As pessoas indiretamente envolvidas - os usuários-finais - perfazem um total de 30. Tratando-se este de um estudo qualitativo, a amostra utilizada, conforme ilustrado na figura 1, é do tipo intencional (TRIVIÑOS 1994). Definiram-se os seguintes critérios para escolha da amostra: representatividade (significa entrevistar pessoas dos três diferentes grupos do universo, dos três campi, e das três áreas da instituição referentes aos módulos implementados do ERP); disponibilidade (significa entrevistar pessoas que estavam presentes na instituição com tempo disponível para a entrevista durante a etapa de coleta de dados) e acessibilidade (significa entrevistar pessoas residentes no estado de Santa Catarina).

Tabela 1: Universo e amostra da pesquisa

\begin{tabular}{l|l|l}
\hline GRUPOS DO UNIVERSO & UNIVERSO & AMOSTRA \\
\hline $\begin{array}{l}\text { Pessoas diretamente envolvidas } \\
\text { (da instituição) }\end{array}$ & 27 & 19 \\
\hline $\begin{array}{l}\text { Pessoas diretamente envolvidas } \\
\text { (da empresa contratada) }\end{array}$ & 10 & 3 \\
\hline $\begin{array}{l}\text { Pessoas indiretamente envolvidas } \\
\text { (da instituição) }\end{array}$ & 30 & 6 \\
\hline & 67 & 28 \\
\cline { 2 - 3 }
\end{tabular}

Fonte: elaborada pelas autoras.

Portanto, a pesquisa contou com um total de 28 participantes assim distribuídos: 19 pessoas da instituição diretamente envolvidas no projeto, 6 usuários-finais que não 
participaram diretamente do projeto e 3 consultores da empresa SAP contratados para o projeto.

No caso das pessoas da instituição, dados primários foram obtidos com base em entrevistas semi-estruturadas (TRIVIÑOS, 1994). As entrevistas, num total de vinte e cinco, foram conduzidas no ambiente de trabalho dos participantes. Além das entrevistas realizadas com os colaboradores da Universidade, foram enviados questionários abertos para os consultores da empresa SAP. Como só houve dois respondentes do questionário, optou-se por enviá-lo novamente obtendo-se mais um respondente, perfazendo um total de três consultores. A fim de complementar as informações fornecidas nas entrevistas e questionários, buscou-se dados secundários por meio de documentos referentes ao projeto, como informativos, jornais internos e publicações no portal da instituição, que pudessem colaborar com informações sobre o tema em questão.

A análise dos dados foi realizada com base em um estudo aprofundado dos dados coletados num processo de idas e vindas da análise desses dados ao referencial teórico apresentado, procurando dar um suporte teórico às reflexões elaboradas. Buscou-se classificar e categorizar os dados nas seguintes categorias - a) implementação do sistema e pessoas envolvidas; b) meios de compartilhamento do conhecimento; c) fatores que influenciaram o compartilhamento - o que proporcionou ao pesquisador maior compreensão do processo de implementação do sistema e de como ocorreu o compartilhamento de conhecimentos nesse processo.

\section{APRESENTAÇÃO E ANÁLISE DOS DADOS}

\subsection{A NATUREZA DO PROJETO E AS PESSOAS ENVOLVIDAS}


O projeto de implementação do SAP R/3 abrangeu os três campi da Universidade e contemplou a integração dos seus processos administrativos e financeiros, com a implementação dos seguintes módulos do sistema: Financeiro (FI), Controladoria (CO) e Materiais (MM). O Projeto desenvolveu-se durante um ano e três meses, dentro do escopo e prazo inicialmente definidos. Pode-se caracterizar o projeto em três grandes períodos genéricos, assim definidos: pré-implantação, implantação e pós-implantação.

No período pré-implantação foi identificada a necessidade de mudança de sistemas na Universidade em decorrência do grande crescimento da instituição em um curto período de tempo e da consequente necessidade de um suporte informacional que proporcionasse uma visão integrada de seus setores e diferentes campi. Foi então constituído um grupo multidepartamental e multicampi o qual realizou um processo metódico de análise de adequação dos sistemas e fornecedores existentes no mercado em relação às necessidades da instituição, culminando com a seleção da empresa SAP e seu sistema SAP R/3. A própria fornecedora do sistema (SAP) fez questão de prestar a maior parte dos serviços de consultoria, isto porque, a Universidade era a primeira do segmento educacional a implantar o R/3. Apresentava-se este caso, para a fornecedora, como uma oportunidade de obter know-how no segmento.

A implantação do sistema, momento a partir do qual passaram a trabalhar em conjunto as pessoas da empresa contratada e as pessoas da Universidade selecionadas para o projeto, foi orientada pela metodologia de implantação da SAP denominada ASAP (Accelerated SAP). A equipe de implantação foi composta por equipes de trabalho, com dedicação full time, estruturadas segundo cada módulo. Essas equipes eram compostas por profissionais ligados às áreas da instituição envolvidas no projeto (usuários-chave), da área de TI e pelos consultores. A posição da gerência do projeto foi ocupada por um executivo da instituição e um da SAP. Foram definidos comitês com dedicação parcial ao projeto: o comitê executivo (representantes da alta-administração da instituição - reitoria e diretores de campi) e o 


\section{MEIOS DE COMPARTILHAMENTO DO CONHECIMENTO NO CONTEXTO DE PROJETOS DE TECNOLOGIA DA INFORMAÇÃO}

Clarissa Carneiro Mussi - Maria Terezinha Angeloni

comitê de validação (diretores das áreas da instituição envolvidas no projeto e dois representantes da área de $\mathrm{TI}$ ). Foi também contratado um consultor cujo papel era de sensibilização dos colaboradores da instituição para as mudanças que acompanham a implementação de um sistema integrado.

No período pós-implantação do sistema, quando a presente pesquisa foi realizada, as atividades de consultoria da empresa SAP haviam sido finalizadas e os usuários encontravam-se em fase inicial de interação e aprendizado do sistema. Alguns ajustes estavam também sendo realizados, em decorrência de mudanças organizacionais que vinham ocorrendo com a troca de reitoria.

\subsection{OS MEIOS DE COMPARTILHAMENTO DO CONHECIMENTO}

Observou-se que, independente do número de etapas do projeto SAP R/3, um número de indivíduos internos e externos à Universidade participaram desse projeto, incorporando-Ihe diferentes conhecimentos, como preconizam Clegg, Waterson e Axtell (1997). Essa questão é destacada por alguns entrevistados quando falam sobre a interação entre os colaboradores da Universidade e os consultores da SAP:

[...] nós sabíamos como funcionavam os processos aqui dentro e a SAP tinha

o know-how, conhecimento, de como funcionava o sistema, de como adaptar esse sistema aos nossos processos. Então foi esse casamento que houve, a SAP entrou com o conhecimento do software efetivamente e nós com o conhecimento dos processos (ent. 22); [...] o consultor, a pessoa de fora tinha o conhecimento do sistema e nós conhecíamos a unidade de gestão, então a gente une os dois para chegar no melhor (ent. 21).

O compartilhamento do conhecimento no projeto configurou-se por meio de um conjunto de recursos (meios de compartilhamento). A seguir, são apresentados estes recursos, seus objetivos e os períodos do projeto em que foram utilizados. 


\section{MEIOS DE COMPARTILHAMENTO DO CONHECIMENTO NO CONTEXTO DE PROJETOS DE TECNOLOGIA DA INFORMAÇÃO}

Clarissa Carneiro Mussi - Maria Terezinha Angeloni

\section{$\Rightarrow$ Metáforas e analogias}

Pôde-se perceber, principalmente no período pré-implantação, a utilização de metáforas e analogias com o objetivo de buscar a aproximação de um contexto com o qual grande parte das pessoas da instituição não tinha experiência ou do qual não tinha conhecimento. Como dizem Nonaka e Takeuchi (1997), metáforas e analogias permitem que indivíduos fundamentados em contextos e experiências diferentes compreendam algo mais facilmente. A metáfora do corpo humano, por exemplo, foi utilizada no compartilhamento da idéia de mudança de uma organização departamental, contexto no qual estava baseada a experiência dos colaboradores da instituição, para uma organização orientada por processos, o que pode ser observado na fala de um dos entrevistados:

[...] a gente tentou passar a idéia de que a prioridade era a Universidade, e não a prioridade é o melhor para o seu departamento. [...] essa idéia não foi muito pacífica. [...] Então, a metáfora que nós fazíamos na ocasião é que a gente era um corpo, e o coração não poderia ter o melhor funcionamento, em detrimento se o fígado, por exemplo, não funcionasse bem. O corpo não iria bem. Então eu não queria o melhor sistema de contabilidade isolado [...], mas que as informações que vinham das outras áreas que ele necessita, viessem numa qualidade e velocidade muito maior. Então, poderia perder em termos de funcionalidade de sistemas isolados, mas ficaria ágil em virtude da integração (ent. 3).

Uma analogia, por exemplo, foi utilizada na seleção das pessoas das áreas/setores da Universidade que iriam participar do projeto em tempo integral. Como o número de pessoas trabalhando em cada área não era muito grande, a saída de algumas pessoas de sua área para participar do projeto era uma questão a ser também compartilhada. Assim relata um entrevistado:

[...] o que nós dissemos na hora de recrutar as pessoas foi que elas seriam demitidas da sua área, iriam trabalhar seis meses no projeto, iriam ser demitidas do projeto e iriam voltar a ser recontratadas pela área. Para o chefe nós dissemos o seguinte: nós vamos pegar o teu funcionário, ficar seis meses com ele e te devolver melhor porque tem um conjunto de 


\section{MEIOS DE COMPARTILHAMENTO DO CONHECIMENTO NO CONTEXTO DE PROJETOS DE TECNOLOGIA DA INFORMAÇÃO \\ Clarissa Carneiro Mussi - Maria Terezinha Angeloni}

treinamento e o fato da experiência de fazer parte de uma equipe é muito importante (ent. 25).

Neste caso, o uso da analogia visava a transmitir a concepção de implementação de um sistema a partir de um projeto, o que exigia que alguns colaboradores deixassem as suas áreas por um período de tempo para participar do projeto e com ele contribuir. Esta também não era uma realidade familiar às experiências de implementação de sistemas na instituição.

\section{$\Rightarrow$ Reuniões}

As reuniões foram extensivamente utilizadas durante o projeto. No período pré-implantação, várias reuniões foram conduzidas com o objetivo de sensibilização para a idéia de mudança. Assim, relata um entrevistado: "[...] tivemos diversas reuniões para sensibilizar para esta idéia, trabalhar esta idéia e este circuito de reuniões serviu para firmar bem esta idéia, então ficamos quase todo mundo num discurso só" (ent. 25). As palavras do entrevistado vão ao encontro do que explica Jay (1999) quando diz que as reuniões, se bem conduzidas, podem auxiliar cada indivíduo a compreender o objetivo do grupo e como seu trabalho individual pode contribuir para atingir esse objetivo. Pode criar nos participantes um compromisso com as decisões tomadas e com os objetivos a serem buscados. Ainda no período préimplantação, durante a avaliação dos sistemas existentes no mercado, foram também realizadas reuniões com o objetivo de estreitar $o$ relacionamento com os fornecedores, conhecer melhor seus sistemas e solucionar dúvidas sobre as respostas dadas aos questionários enviados pela Universidade relativos à adequação dos sistemas às necessidades da instituição. Na etapa de implantação do sistema, mesmo as equipes de trabalho estando estruturadas por módulo, em muitos momentos, toda a equipe do projeto era reunida para discussão dos processos como um todo. Eram também realizadas reuniões periódicas com o comitê executivo, para relatar o andamento do projeto, e com comitê de validação, a fim de validar as adequações que estavam sendo feitas no sistema. 


\section{$\Rightarrow$ Questionários e entrevistas}

$\mathrm{Na}$ fase de avaliação e seleção dos sistemas existentes no mercado foi elaborado um tipo de questionário - o RFI (Request for Information) - que tinha como objetivo o levantamento de informações sobre as empresas fornecedoras e seus sistemas, especialmente no que diz respeito à adequação do sistema às necessidades da Universidade. Para a elaboração do questionário foram feitas entrevistas com alguns colaboradores das diferentes áreas de forma a identificar as necessidades de cada área. Na etapa de implantação do sistema, uma das formas de os consultores conhecerem os procedimentos e práticas da instituição foi a partir de entrevistas realizadas com os integrantes do projeto. Nessas entrevistas foi utilizado um questionário padrão, previsto pela metodologia ASAP, que após respondido, servia de balizador para a parametrização e implantação do sistema. Um entrevistado explica:

[...] a metodologia ASAP previa umas perguntas e a gente ia respondendo.

Como por exemplo: 'quantos campi tem a Universidade?', 'o processo de compras vai ter alguém liberando?'. Então já provoca o grupo com questões, criando uma situação que tenda a mostrar a Universidade para aquele consultor que está vindo de fora [...] (ent. 26).

\section{$\Rightarrow$ Conversação face a face}

Os entrevistados foram unânimes em ressaltar a conversação face a face como meio de interação com os consultores. Quando indagados sobre os meios utilizados para compartilhar os conhecimentos seja do sistema, seja das práticas organizacionais da Universidade, os entrevistados explicam: [...] era lado a lado (ent. 20). [...] na frente do computador, mexendo e mostrando e eu anotando tudo (ent. 21). [...] conversando, discutindo, se dava algum problema, a gente já ia ali, sentava, discutia e tentava resolver [...] foi um processo bem interativo (ent. 4). Na interação entre consultores e equipe da Universidade, os entrevistados comentam que a inquirição era bastante utilizada como um forma de suprir as diferenças de linguagem 
entre ambas as partes. Como afirma o entrevistado 20: "a gente perguntava, 'o que tu queres dizer com isso?', 'o que isso quer dizer?' aí eles diziam, 'não isso aqui é' [...]" (ent. 11). Foi possível observar que a inquirição foi utilizada especialmente pela equipe da Universidade nas conversações com os consultores como forma de estabelecer uma linguagem de entendimento comum entre ambas as partes e, como dizem Senge et al (1999), como um meio de buscar compreender o pensamento e raciocínio do outro.

\section{$\Rightarrow$ Simulações}

Nas simulações, dados reais da empresa são passados ao sistema e, por meio de tentativas, erros e acertos, conhecimentos são compartilhados buscando-se identificar as adequações que necessitam ser feitas. Para Chew, Leonard-Barton, e Bohn (1991), as simulações são úteis quando se deseja conhecer, mesmo que parcialmente, a interação de um sistema com a realidade organizacional. As simulações foram bastante utilizadas na etapa de implantação, especialmente quando da adequação do sistema à instituição. Vários entrevistados ressaltaram o aprendizado do sistema por meio da simulação. Como explica o entrevistado 11: [...] a gente testava, ia ver o que afetava, a gente mudava uma coisinha em Materiais, 'ah, vamos ver o que isso afeta lá no Financeiro', 'o que afeta em contabilidade', via o processo na íntegra [...]. Um dos consultores entrevistados (ent. 28) também comenta que a "replicação de cenários no ambiente de testes, os testes integrados" foi uma forma de os usuários participantes do projeto adquirirem conhecimentos operacionais sobre o sistema.

\section{$\Rightarrow$ Treinamento prático}

No início do período de implantação, antes de proceder à adequação do sistema à empresa, foram realizados treinamentos pelo fornecedor do sistema e consultores para os usuários-chave. Neste primeiro treinamento foram expostas as funcionalidades gerais do sistema e de cada módulo, com a finalidade de os 
participantes do projeto (usuários-chave) conhecerem o sistema e participarem mais ativamente do processo de adequação do sistema à empresa. No final da etapa de implantação, foi conduzido o treinamento do sistema para os usuários-finais pelos usuários-chave, neste caso não houve a participação da SAP. O objetivo dos treinamentos realizados, tanto para os usuários-chave quanto para os usuários-finais, era transmitir conhecimentos relativos à operação do sistema.

\section{$\Rightarrow$ Workshops}

$\mathrm{Na}$ etapa de implantação do sistema, foram realizados workshops de sensibilização para a mudança, conduzidos por um consultor contratado especificamente para esse fim. 0 entrevistado 27 explica que o objetivo dos workshops era "preparar as pessoas para entender que mudanças iriam ocorrer junto com a implementação do SAP". As pessoas da Universidade que participaram dos workshops foram a equipe de projeto e alguns gestores. Embora alguns entrevistados utilizem o termo "palestra" para se referirem aos encontros realizados pelo consultor, pôde-se perceber que esses encontros assumiam características mais de workshops do que de palestras, considerando que havia uma interação entre ambos, consultor e participantes, como comentam alguns entrevistados: "foi um grupo assim de batepapo, interagindo" (ent. 22); "nós conversamos sobre mudanças, como acontecia nas outras organizações [...]" (ent. 25).

\section{$\Rightarrow$ Visitas e contatos com material e pessoas especializadas}

No período pré-implantação, com o objetivo de suscitar na Universidade o compartilhamento de questões relacionadas a projetos ERP, foram utilizados instrumentos como textos e livros, conforme comenta o entrevistado 25: "a gente trouxe diversos textos sobre integração de sistemas, mudança de cultura, um livro com título Peopleware que foi comprado para toda a equipe e debatido alguns pontos do livro [...]". Em adição, a fim de obter conhecimentos sobre sistemas ERP, antes de sua 
adoção, foram realizadas visitas a empresas que já haviam implementado tais sistemas. Também, após o R/3 ter sido implementado na Universidade (período pósimplantação), foram realizadas visitas e troca de experiências com outras empresas que utilizam o sistema do mesmo fornecedor. 0 entrevistado 20 relata: "[...] a gente mantém contato com empresas que têm sistemas ERP, e busca informações que possam nos ajudar [...] fomos visitar uma empresa que tinha implantado o $R / 3$ para nós tirarmos algumas dúvidas [...] nós buscamos, diante das nossas dificuldades, questionar sobre como funcionava na empresa deles". Os contatos e visitas a empresas que já haviam implantado o sistema, conforme relatado pelo entrevistado, permitiram o compartilhamento de experiências.

\section{$\Rightarrow$ Jornal e home page da universidade}

Para as pessoas que não trabalharam diretamente no projeto, o jornal interno e mensal da Universidade e uma home page sobre o projeto mostraram-se úteis como meios de compartilhamento de informações sobre o projeto e seu andamento, de forma a manter os colaboradores da instituição informados. Assim descreve o entrevistado 25:

[...] para as pessoas da organização que ouviram falar no projeto, não estavam diretamente ligadas, não foram chamadas a trabalhar porque também não envolvia a sua área, ou também que envolvia a sua área, mas estavam trabalhando mais porque o colega saiu, a home page foi criada para divulgar, e notícias no jornal da universidade. A universidade tem um jornal que circula mensalmente e durante a implementação do projeto todos os jornais tinham alguma coisa com relação ao projeto, andamento, status, etc.

\section{$\Rightarrow$ A documentação}

Todas as reuniões realizadas ao longo do projeto foram registradas em atas e todas as decisões tomadas foram documentadas, como comentam os entrevistados: 


\section{MEIOS DE COMPARTILHAMENTO DO CONHECIMENTO NO CONTEXTO DE PROJETOS DE TECNOLOGIA DA INFORMAÇÃO}

Clarissa Carneiro Mussi - Maria Terezinha Angeloni

[...] nós fazíamos o registro de todas as nossas reuniões e as abordagens feitas, foram emitidos questionários aos fornecedores, todos foram registrados, toda a parte impressa que foi produzida pela equipe foi mantida em arquivo (ent. 17); [...] havia comitês para as tomadas de decisão e todas as decisões tomadas ao longo do projeto com o consultor [...] foram documentadas, devidamente arquivadas, registradas formalmente. Isso tem até hoje. Então, qualquer modificação, qualquer decisão que você venha a tomar hoje, facilmente você consegue ir lá e saber por que foi tomada essa decisão (ent. 18).

Na etapa de implantação, foram utilizados repositórios de conhecimento, disponíveis a toda a equipe, que mantinham os documentos de cada módulo, resultantes das atividades do projeto, facilitando o compartilhamento e fornecendo uma visão centralizada do projeto. Esta documentação foi útil para o compartilhamento das atividades e experiências não só durante o projeto, mas também estava servindo como base de conhecimento para a empresa, depois que o projeto foi finalizado. Os entrevistados destacam essa questão:

[...] qualquer pessoa que queira saber como foi o processo de implantação, tem tudo documentado (ent 6); este conhecimento fica registrado no sentido de que quando vier outra pessoa, não precisa eu sair e levar o conhecimento comigo, está lá registrado, o que foi feito, o que não foi feito, como funciona, como não funciona (ent. 22).

Quanto aos conhecimentos obtidos sobre a operação do sistema, a documentação foi feita pelos próprios usuários-chave. Cada equipe de trabalho documentava os conhecimentos obtidos de seu módulo, à medida que eram transmitidos pelos consultores. Esses conhecimentos eram passados para documentos, previstos pela metodologia ASAP, que consistiam em um manual de como executar uma determinada transação, operação.

\section{$\Rightarrow$ A tecnologia da informação}

$\mathrm{Na}$ etapa de implantação, um dos conhecimentos explícitos que necessitou ser compartilhado e entendido por todos os participantes do projeto foi a própria metodologia de implantação do sistema, no caso a ASAP. Foi utilizado o software 
Microsoft Project, justamente com esse objetivo. Os documentos de cada módulo relativos ao projeto estavam disponíveis e eram compartilhados entre todos os envolvidos. Como diz o entrevistado 18, "cada grupo com o respectivo consultor tinha sua documentação própria de cada fase do projeto. Eles tinham documentos que a própria metodologia trazia". Redes de computadores e estruturas de arquivos também foram empregados para armazenamento e acesso da documentação, por toda a equipe, dos produtos gerados de cada fase do projeto. O Microsoft Word foi utilizado para registro da documentação da operação do sistema feita pelas equipes de trabalho durante o contato com os consultores, estando hoje disponível para todos os usuários.

\section{$\Rightarrow$ O Help Desk da SAP e os Grupos de Usuários}

O Help Desk da SAP, suporte virtual oferecido pela SAP após a implantação do sistema, foi utilizado pela equipe da Universidade que participou do projeto para trocar algumas informações e solucionar dúvidas. Os entrevistados também se referiram ao acesso do site da própria SAP como um meio de obter informações. Alguns entrevistados comentaram a participação em grupos de usuários do R/3 e em listas de discussão, como um meio de compartilhamento de experiências, de esclarecimento de dúvidas e de aquisição de novos conhecimentos, o que pode ser observado no depoimento de um deles: "[...] eu tenho procurado manter contato com o pessoal de uma lista de discussão de todo Brasil [...] a lista para mim é uma maravilha. Tudo que eu acho, se eu penso em colocar isso no sistema, eu pergunto 'oh, o que vocês acham?' E vêm várias respostas" (ent.12 ).A participação de grupos de usuários, listas de discussão, não apenas do software em si, mas também de questões relacionadas a sistema integrados, às mudanças que esses sistema podem trazer, pode ser um meio de compartilhamento de experiências, de esclarecimento de dúvidas e de aquisição de novos conhecimentos. Como observa Hehn (1999), após a implantação do sistema é importante estar continuamente aprendendo tanto sobre o que foi instalado quanto sobre as novidades que surgem. Conforme o autor, esses conhecimentos 


\section{MEIOS DE COMPARTILHAMENTO DO CONHECIMENTO NO CONTEXTO DE PROJETOS DE TECNOLOGIA DA INFORMAÇÃO}

Clarissa Carneiro Mussi - Maria Terezinha Angeloni

podem ser adquiridos por meio de treinamento, mas também dependerão do interesse e esforço de cada um.

Como pode ser observado, na análise do projeto ERP na Universidade, identificaram-se vários meios pelos quais os conhecimentos foram compartilhados. Dentre esses meios encontram-se tanto aqueles que permitem o compartilhamento do conhecimento explícito, como por exemplo, a documentação (eletrônica ou não), o jornal e home page da instituição, quanto aqueles com potencial para o compartilhamento do conhecimento tácito, como por exemplo, a conversação face a face, os workshops de sensibilização, as simulações.

Os meios de compartilhamento do conhecimento utilizados também propiciaram os processos de conversão do conhecimento definidos por Nonaka e Takeuchi (1997). A figura 2 apresenta os meios de compartilhamento do conhecimento anteriormente abordados, seus objetivos e os processos de conversão do conhecimento que propiciaram.

Quadro 1: Meios de compartilhamento do conhecimento no Projeto.

\begin{tabular}{|c|c|c|}
\hline $\begin{array}{l}\text { MEIO } \\
\text { DE COMPARTILHA- } \\
\text { MENTO }\end{array}$ & OBJETIVO & $\begin{array}{l}\text { CONVERSÃO DO } \\
\text { CONHECIMENTO }\end{array}$ \\
\hline $\begin{array}{l}\text { Metáforas } \\
\text { Analogias }\end{array}$ & $\begin{array}{l}\text {. Buscar a aproximação de um contexto com o qual grande parte das } \\
\text { pessoas da instituição não tinha experiência ou do qual não tinha } \\
\text { conhecimento. } \\
\text { Metáfora do corpo humano: visão centrada em processos. } \\
\text { Analogia recrutamento e seleção: concepção da implementação de um } \\
\text { sistema por meio de um projeto. }\end{array}$ & Externalização \\
\hline Reuniões & $\begin{array}{l}\text {. Sensibilizar para a idéia de mudança buscando uma visão comum. } \\
\text {. Estreitar o relacionamento e solucionar dúvidas com os fornecedores. } \\
\text { - Apresentar produtos de cada fase do projeto e tomar decisões. } \\
\text { - Validar as decisões tomadas (comitê de validação) e relatar o } \\
\text { andamento do projeto (comitê executivo). }\end{array}$ & Combinação \\
\hline $\begin{array}{l}\text { Questionários e } \\
\text { Entrevistas }\end{array}$ & $\begin{array}{l}\text { - Levantar informações sobre as empresas fornecedoras e seus } \\
\text { sistemas (questionário). } \\
\text { - Identificar as necessidades e características de cada área (entrevistas } \\
\text { com colaboradores das diferentes áreas). } \\
\text { - Transmitir conhecimentos aos consultores sobre os procedimentos e } \\
\text { práticas da Universidade (entrevistas) }\end{array}$ & $\begin{array}{l}\text { Externalização } \\
\text { Combinação }\end{array}$ \\
\hline $\begin{array}{l}\text { Conversação face } \\
\text { a face }\end{array}$ & $\begin{array}{l}\text { - Trocar conhecimentos sobre o sistema e práticas de trabalho da } \\
\text { Universidade (consultores e as equipes de trabalho). Presença da }\end{array}$ & $\begin{array}{l}\text { Socialização } \\
\text { Externalização }\end{array}$ \\
\hline
\end{tabular}




\section{MEIOS DE COMPARTILHAMENTO DO CONHECIMENTO NO CONTEXTO DE PROJETOS DE TECNOLOGIA DA INFORMAÇÃO}

Clarissa Carneiro Mussi - Maria Terezinha Angeloni

\begin{tabular}{|c|c|c|}
\hline & $\begin{array}{l}\text { inquirição como uma forma de estabelecer uma linguagem de } \\
\text { entendimento comum entre ambas as partes. }\end{array}$ & $\begin{array}{l}\text { Combinação } \\
\text { Internalização }\end{array}$ \\
\hline Simulações & $\begin{array}{l}\text { - Aprendizado do sistema, testes e adequação do sistema à } \\
\text { Universidade. }\end{array}$ & Internalização \\
\hline $\begin{array}{l}\text { Treinamento } \\
\text { prático }\end{array}$ & $\begin{array}{l}\text { - Transmissão de conhecimentos sobre a operação do sistema aos } \\
\text { usuários-chave pelos consultores. Transmissão de conhecimentos da } \\
\text { operação do sistema aos usuários-finais pelos usuários-chave. }\end{array}$ & $\begin{array}{l}\text { Socialização } \\
\text { Internalização }\end{array}$ \\
\hline Workshops & $\begin{array}{l}\text { - Preparar as pessoas (reitoria, gerentes, equipe de projeto) para } \\
\text { entender que mudanças iriam ocorrer com a implementação do } \\
\text { sistema. }\end{array}$ & $\begin{array}{l}\text { Socialização } \\
\text { Combinação }\end{array}$ \\
\hline $\begin{array}{l}\text { Visitas e contato } \\
\text { com material e } \\
\text { pessoas } \\
\text { especializadas }\end{array}$ & $\begin{array}{l}\text { - Utilização de textos e livros para suscitar o compartilhamento de } \\
\text { questões relacionadas a sistemas integrados e mudança } \\
\text { organizacional. } \\
\text { - Obtenção de maior conhecimento sobre sistemas integrados, } \\
\text { esclarecimento de dúvidas e troca de experiências (visitas a outras } \\
\text { empresas) }\end{array}$ & $\begin{array}{l}\text { Combinação } \\
\text { Internalização }\end{array}$ \\
\hline $\begin{array}{l}\text { Jornal e home } \\
\text { Page }\end{array}$ & $\begin{array}{l}\text {. Divulgação e transmissão de informações sobre a implantação para os } \\
\text { usuários que não participaram diretamente do projeto e demais } \\
\text { colaboradores da Universidade. }\end{array}$ & Combinação \\
\hline Documentação & $\begin{array}{l}\text {. Registro eletrônico das atas de reuniões, registro de documentos de } \\
\text { contato com os fornecedores e das decisões tomadas. } \\
\text {. Facilitar o compartilhamento e fornecer uma visão centralizada do } \\
\text { projeto (repositórios de conhecimento). }\end{array}$ & $\begin{array}{l}\text { Externalização } \\
\text { Internalização } \\
\text { Combinação }\end{array}$ \\
\hline $\begin{array}{l}\text { Tecnologia } \quad \text { da } \\
\text { informação }\end{array}$ & $\begin{array}{l}\text {. Representação detalhada da metodologia e dos produtos das etapas } \\
\text { no Microsoft Project fornecendo a toda equipe uma visão centralizada } \\
\text { do projeto } \\
\text { - Registro no Microsoft Word dos conhecimentos obtidos sobre a } \\
\text { operação do sistema pelos usuários-chave, disponível a todos os } \\
\text { usuários para consulta pós-implementação.. }\end{array}$ & $\begin{array}{l}\text { Internalização } \\
\text { Externalização } \\
\text { Combinação }\end{array}$ \\
\hline $\begin{array}{l}\text { Help Desk SAP e } \\
\text { os Grupos de } \\
\text { usuários }\end{array}$ & $\begin{array}{l}\text { Suporte virtual SAP utilizado pela equipe do projeto para solucionar } \\
\text { dúvidas } \\
\text {. Troca de experiências e busca de informações e conhecimentos }\end{array}$ & $\begin{array}{l}\text { Socialização } \\
\text { Combinação } \\
\text { Internalização }\end{array}$ \\
\hline
\end{tabular}

Fonte: elaborada pelas autoras.

A figura 2 mostra que os quatro modos de conversão do conhecimento apareceram no contexto do projeto propiciados pelos meios de compartilhamento do conhecimento identificados, o que reforça a relação entre compartilhamento e criação do conhecimento. A seguir apresentam-se as considerações finais da pesquisa.

\section{CONSIDERAÇÕES FINAIS}




\section{MEIOS DE COMPARTILHAMENTO DO CONHECIMENTO NO CONTEXTO DE PROJETOS DE TECNOLOGIA DA INFORMAÇÃO \\ Clarissa Carneiro Mussi - Maria Terezinha Angeloni}

Ao finalizar o estudo do projeto de implementação do SAP R/3 na Universidade, considera-se que algumas questões merecem destaque. Uma delas relaciona-se à experiência que a instituição passou ao "trabalhar" a implementação do sistema por meio de um projeto. O Projeto teve um orçamento próprio, um escopo definido e um prazo a ser cumprido. Esta não era uma experiência familiar às implementações de sistemas na instituição que ocorriam no âmbito dos próprios departamentos. Assim, parece que a própria forma de trabalhar por projeto (o trabalho em equipe, a interação interdepartamental, a necessidade do compartilhamento) já contribuiu para que seus participantes começassem a incorporar a filosofia de trabalho que um sistema integrado requer.

Foi possível perceber que a definição dos meios pelos quais o conhecimento é compartilhado e a forma com que esses meios são utilizados podem facilitar e/ou dificultar o compartilhamento e conseqüentemente a implementação do sistema. A aquisição de conhecimentos operacionais sobre o sistema (por meio do treinamento) pela equipe da Universidade, antes de iniciar a implantação, é um exemplo da opção de utilização de um meio de compartilhamento em um determinado momento que constituiu um fator facilitador.

Destaca-se a ênfase dada pela instituição aos meios potenciais de compartilhamento do conhecimento tácito, notadamente durante a implantação do sistema na interação entre consultores e equipe da Universidade. Nesse período predominaram a conversação face a face e meios que permitiram o aprender-fazendo como, por exemplo, as simulações pelas quais eram realizados testes integrados do sistema com os dados "reais" da instituição. Este é um fator positivo considerando, nesse período, a necessidade de um processo intensivo de compartilhamento de forma que os consultores obtivessem a visão dos processos da instituição e a equipe Universidade, a visão das funcionalidades do sistema. Em contrapartida, o fato de a instituição ser a primeira organização universitária a implementar o R/3, de certa 
forma dificultou o compartilhamento e o projeto, já que os consultores não haviam passado por experiências nesse tipo de segmento.

Embora se tenha observado vários meios potenciais de compartilhamento do conhecimento tácito, estes parecem ter sido utilizados especialmente "dentro do projeto" - entre equipe da Universidade que participou diretamente do projeto e consultores. As práticas e meios de compartilhamento do conhecimento "fora do projeto" foram fracas e não sistemáticas. Não foi estabelecido um processo sistematizado de interação e integração da equipe do projeto com os demais usuários do sistema, o que poderia ter propiciado um compartilhamento mais efetivo entre ambas as partes, principalmente quanto às necessidades das áreas envolvidas.

Quanto ao compartilhamento dos conhecimentos relacionados ao sistema entre consultores e equipe da Universidade, os depoimentos dos entrevistados revelam ter havido maior preocupação com a incorporação dos conhecimentos sobre a operação do sistema (know-how), e menor ênfase aos conhecimentos relativos a sua parametrização (know-why). Ambos os conhecimentos são importantes e complementam-se (KIM, 1993). Este caso de parcialidade do conhecimento, como denominam Clegg et al (1997), dificultou a visualização pelos colaboradores da instituição da melhor combinação de parâmetros do sistema que reflita o contexto da instituição e as mudanças que nele ocorrem e pode indicar uma maior dependência de pessoas externas quando da necessidade deste tipo de conhecimento.

A busca pela troca de experiências com empresas que já implementaram sistemas integrados, por meio de visitas e o contato com material especializado (leitura de livros e artigos sobre sistemas ERP) nos períodos pré e pós-implantação, também merecem destaque, considerando que esta foi uma oportunidade para aquisição de conhecimentos. Ressalta-se ainda a preocupação com a documentação do projeto de implementação do sistema, o que está sendo útil para consultas, para o aprendizado de novos colaboradores da instituição e para auxílio em outros projetos. 
Ainda que o contexto de projetos de $\mathrm{TI}$, especialmente de projetos ERP, tenda a ser influenciado por inúmeros fatores, as evidências deste estudo reforçam a importância de observar e "trabalhar" aqueles relacionados ao compartilhamento do conhecimento, visando a buscar a efetividade do projeto.

Por fim, entende-se que o tema abordado poderá suscitar futuras pesquisas como, estudos de caso comparativos dentro da investigação proposta; análise de projetos de TI com foco em outros processos da gestão do conhecimento como, por exemplo, a criação e a codificação do conhecimento.

\section{MEANS OF KNOWLEDGE SHARING IN INFORMATION TECHNOLOGY PROJECTS}

\section{ABSTRACT}

This study tried to investigate in what ways the knowledge sharing among the parts involved in the implementation project of an integrated information system (SAP R/3) in a higher education institution of the Santa Catarina State was configured. Specifically, the work obeyed the following disposition: description of how the implementation process occurred and identification of the individuals involved in it; investigation of the means of knowledge sharing utilized, objectives and influence in the project. Considering the objectives proposed, the approach of this research is qualitative, case study type. The research data were collected through semi-structured interviews, open questionnaires and documental analysis. The treatment and interpretation given to the data are predominantly qualitative. Among the main conclusions we may cite the significant influence of knowledge sharing in the project of the system implementation. We can observe the utilization of various potential means of sharing both explicit and tacit knowledge that contributed to the knowledge 
MEIOS DE COMPARTILHAMENTO DO CONHECIMENTO NO CONTEXTO DE PROJETOS DE TECNOLOGIA DA INFORMAÇÃO

Clarissa Carneiro Mussi - Maria Terezinha Angeloni

conversion. Finally the data analysis provided the understanding that the definition of the means by which the knowledge is shared and the way these means are utilized may, on one hand, facilitate and, on the other, make difficult the knowledge sharing and, consequently, the project of the system implementation.

Keywords: information technology, knowledge sharing, ERP, projects.

\section{REFERÊNCIAS}

BRESNEN, M. et al. Social practices and the management of knowledge in project environments. International Journal of Project Management, v. 21, p. 157-166, 2003.

CHEW, W. B., LEONARD-BARTON, D., BOHN, R. E. Beating murphy's law. Sloan Management Review, v. 32, n. 3, p. 5-16, Spring 1991.

CLEGG, C. W.; WATERSON, P. E.; AXTELL, C. M. Software development: some critical views. Behaviour \& Information Technology, v. 16, n. 6, p. 359-362, 1997.

CRAWFORD, L. Profiling the competent project manager. In: Project Management Research at the Turn of the Millenium: Proceedings of PMI Research Conference, p.315, Paris, France, 21-24 Jun., 2000.

DAVENPORT, T. H. Putting the enterprise into the enterprise system. Harvard Business Review, Boston, v. 76, n. 4, p. 121-131, Jul./Aug. 1998.

DAVENPORT, T. H.; PRUSAK, L. Conhecimento empresarial: como as organizações gerenciam o seu capital intelectual. Rio de Janeiro: Campus, 1998.

HEHN, H. F. Peopleware: como trabalhar o fator humano nas implementações de sistemas integrados de informação (ERP). São Paulo: Gente, 1999.

HUNG, W. H. et al. Relationship bonding for a better knowledge transfer climate: An ERP implementation research. Decision Support Systems, doi:10.1016/j.dss.2011.09.007, 2011. 
MEIOS DE COMPARTILHAMENTO DO CONHECIMENTO NO CONTEXTO DE PROJETOS DE TECNOLOGIA DA INFORMAÇÃO

Clarissa Carneiro Mussi - Maria Terezinha Angeloni

JASIMUDDIN, S. M. A holistic view of knowledge management strategy. Journal of Knowledge

Management, v. 12, n. 2, p. 57-66, 2008.

JAY, A. Como conduzir uma reunião. In: Comunicação eficaz na empresa: como melhorar o fluxo de informações para tomar decisões corretas. Rio de Janeiro: Campus, 1999.

JONES, M. Tacit knowledge sharing during ERP implementation: a multi-site case study. Information Resource Management Journal, v. 18, n. 2, p.1-23, 2005.

KARAPETYAN, A., OTIENO, R. A Study of Knowledge Management Challenges in Project Management: Case of Start-up Projects in Swedish Incubators. 2011. $87 \mathrm{f}$. Masters in Strategic Project Management (European) (Master) - Umea Business School, Umea University, Umea, Sweden, 2011. Disponível em:

<http://urn.kb.se/resolve?urn=urn:nbn:se:umu:diva-39983>. Acesso em: 18 out. 2011.

KARLSEN, J. T., GOTTSCHALK, P. Factors affecting knowledge transfer in IT projects. Engineering Management Journal, v. 16, n.1, p. 3-10, Mar. 2004.

KASVI, J. J. J., VARTIAINEN, M., HAILIKARI, M. Managing Knowledge and knowledge competences in projects and project organizations. International Journal of Project Management, v. 21, p. 571-582, 2003.

$\mathrm{KIM}, \mathrm{D}$. H. The link between individual and organizational learning. Sloan Management Review, v. 35, n.1, p.37-45, Fall 1993.

LAHTI, R. K. Knowledge transfer an management consulting: a look at "the firm".

Business

Horizons, Jan. 2000.

MABERT, V. A. Enterprise Resource Planning: common myths versus evolving reality. Business Horizons, v.44, n.3, p. 69-76, May/June, 2001.

MUSSI, Clarissa. O compartilhamento do conhecimento no processo de implementação de sistemas integrados de informação: o caso da Universidade do Sul de Santa Catarina - Unisul. Florianópolis, 2002. 182p. Dissertação (Mestrado em Administração) - Curso de Pós-Graduação em Administração, Universidade Federal de Santa Catarina.

NONAKA, I e TAKEUCHI, H. Criação de conhecimento na empresa: como as empresas japonesas geram a dinâmica da inovação. Rio de Janeiro: Campus, 1997. 
MEIOS DE COMPARTILHAMENTO DO CONHECIMENTO NO CONTEXTO DE PROJETOS DE TECNOLOGIA DA INFORMAÇÃO

Clarissa Carneiro Mussi - Maria Terezinha Angeloni

NONAKA, I., VON KROGH, G. Tacit knowledge and knowledge conversion: controversy and advancement in organizational knowledge creation theory. Organization Science, v.20, n. 3, p. $635-652$.

PROJECT MANAGEMENT INSTITUTE (PMI). Um Guia do Conhecimento em Gerenciamento de Projetos (Guia $\mathrm{PMBOK}^{\circledR}$ ), 4.ed. Pennsylvania, EUA, 2008. QUINN, J.; ANDERSON, P.; FINKELSTEIN, S. Gerenciando o intelecto profissional: obtendo o máximo dos melhores. In: In KLEIN, D. A. A gestão estratégica do capital intelectual: recursos para a economia baseada em conhecimento. Rio de Janeiro: Qualitymark, 1998. p. 251-286.

REICH, B. H. Managing Knowledge and Learning in IT Projects: a Conceptual Framework and Guidelines for Practice. Project Management Journal, v. 38, n. 2, p. 5-17, 2007.

RUUSKA, I., VARTIAINEN, M. Critical project competences: a case study. Journal of workplace learning, v. 15, n. 7/8, p. 307-312, 2003.

SENG, C. V., ZANNES, E., PACE, R. W. The contributions of knowledge management to workplace learning. Journal of workplace learning, v. 14, n. 4, p. 138-147, 2002.

SENGE, P. et al. A quinta disciplina: caderno de campo: estratégias e ferramentas para construir uma organização que aprende. Rio de Janeiro: Qualitymark, 1999.

SOH, C.; KIEN, S. S.; TAY-YAP, J. Cultural fits and misfits: is ERP a universal solution? Communications of the ACM, Washington DC, v. 43, n. 4, p. 47-51, abr. 2000.

STRAUSS, A., CORBIN, J. Basics of qualitative research: techniques and procedures for developing grounded theory. 3. ed. Thousand Oaks, CA: Sage Publications, 2008.

SVEIBY, K. E. A nova riqueza das organizações: gerenciando e avaliando patrimônios do conhecimento. Rio de Janeiro: Campus, 1998.

TESCH, D. et al. User and developer common knowledge: Effect on the success. International Journal of Project Management, v. 27, p.657-664, 2009.

TRIVIÑOS, A. N. S. Introdução à pesquisa em ciências sociais: a pesquisa qualitativa em educação. São Paulo: Atlas, 1994.

VANDAIE, R. The role of organizational knowledge management in successful ERP implementation projects. Knowledge-based Systems, v. 21, p.920-926, 2008. 
MEIOS DE COMPARTILHAMENTO DO CONHECIMENTO NO CONTEXTO DE PROJETOS DE TECNOLOGIA DA INFORMAÇÃO

Clarissa Carneiro Mussi - Maria Terezinha Angeloni

WANG, E. T. G. et al. Improving enterprise resource planning (ERP) fit toorganizational process through knowledge transfer. International Journal of Information Management, v. 27, p.200-212, 2007.

YIN, R. K. Case study research: design and methods. 4. ed. Thousand Oaks, CA: Sage Publications, 2009. 